\title{
Non-Hermitian Degeneracies and Unidirectional Reflectionless Atomic Lattices
}

\author{
Jin-Hui Wu, ${ }^{1}$ M. Artoni, ${ }^{2,3}$ and G. C. La Rocca ${ }^{4}$ \\ ${ }^{1}$ Center for Quantum Sciences, Northeast Normal University, Changchun 130117, China \\ ${ }^{2}$ European Laboratory for Nonlinear Spectroscopy, 50019 Firenze, Italy \\ ${ }^{3}$ Department of Engineering and Information Technology and INO-CNR Sensor Lab, Brescia University, 25133 Brescia, Italy \\ ${ }^{4}$ Scuola Normale Superiore and CNISM, 56126 Pisa, Italy \\ (Received 8 April 2014; published 19 September 2014)
}

\begin{abstract}
Light propagation in optical lattices of driven cold atoms exhibits non-Hermitian degeneracies when the first-order modulation amplitudes of real and imaginary parts of the probe susceptibility are manipulated to be balanced. At these degeneracies, one may observe complete unidirectional reflectionless light propagation. This strictly occurs with no gain and can be easily tuned and fully reversed as supported by the transfer-matrix calculations and explained via a coupled-mode analysis.
\end{abstract}

DOI: 10.1103/PhysRevLett.113.123004

Much attention has been devoted to the development of artificial metamaterials for achieving optical functionalities not available in nature. Photonic crystals [1] and lefthanded materials [2] are prominent instances tailored to stretch the rules of light propagation and interaction. Such metamaterials have seeded new paradigms in optical, optoelectronic, and optomechanical devices [3-6]. Nevertheless, some tasks are more difficult than others with unidirectional light transport being a most pronounced example. Significant progress has been made in recent years by developing optical materials with parity-time $(P T)$ symmetry to attain unidirectional light invisibility [7-16]. $P T$-symmetric metamaterials require a delicate balance of gain and loss whereby the complex refraction index satisfies $n(z)=n^{*}(-z)$ and are typically made of periodic solid microstructures. Homogeneous atomic vapors driven into three-level [17,18] or four-level [19] configurations have also been proposed to realize $P T$-symmetric optical potentials via rather complicated spatial modulations of two driving fields. Such proposals have obvious advantages of real-time all-optical reconfigurable capabilities and implicit disadvantages of intractable field modulations and considerable symmetry errors. Large optical nonreciprocities may also be achieved by exploiting the asymmetric Doppler shift in moving atomic Bragg mirrors [20], and proofof-principle experiments have been carried out [21].

The great interest in $P T$-symmetric complex media stemmed, however, from the non-Hermitian extensions of quantum mechanics and quantum field theories [22,23], and it is perhaps worth going back to the essential nonHermitian behavior of light transport to get a broader picture on reciprocity violations and unidirectional reflectionlessness. Take, e.g., a typical one-dimensional (1D) light scattering process as shown in Fig. 1(a) where the outgoing field amplitudes $\left\{E_{L}^{-}, E_{R}^{+}\right\}$are related to the incoming field amplitudes $\left\{E_{R}^{-}, E_{L}^{+}\right\}$by a scattering matrix $S$ [24], the eigenvectors of which are defined by
PACS numbers: 37.10.Jk, 11.30.Er, 42.25.Bs, 42.50.Gy

$$
\left(\begin{array}{c}
E_{L}^{-} \\
E_{R}^{+}
\end{array}\right)=\left(\begin{array}{c}
t, r_{L} \\
r_{R}, t
\end{array}\right)\left(\begin{array}{c}
E_{R}^{-} \\
E_{L}^{+}
\end{array}\right)=\lambda_{s}\left(\begin{array}{c}
E_{R}^{-} \\
E_{L}^{+}
\end{array}\right)
$$

The complex amplitudes $t, r_{L}$, and $r_{R}$ of the $(S)$ matrix in Eq. (1) denote, as usual, the reciprocal transmission and the reflection for incidence from the left and from the right. In general, the matrix $S$ is non-Hermitian, its eigenvalues $\lambda_{s}^{ \pm}=t \pm \sqrt{r_{L} r_{R}}$ are complex, and its eigenvectors $\left( \pm \sqrt{r_{L} / r_{R}}, 1\right)^{T}$ are not orthogonal. In particular, they coalesce at a degeneracy, which is at variance with the case of Hermitian degeneracies. This hinges on the fact that for a non-Hermitian matrix, whether symmetric or not, degeneracies are of codimension two. There are two degenerate eigenstates in Eq. (1), representing scattering states with full unidirectional reflectionless propagation from the left $\left(E_{L}^{-}=0, E_{R}^{+} \neq 0\right)$ and from the right $\left(E_{R}^{+}=0\right.$, $E_{L}^{-} \neq 0$ ). Photonic media exhibiting such non-Hermitian degeneracies offer then a unique opportunity to control the unidirectional reflection of light.

We study here a 1D Bragg grating where full unidirectional reflectionlessness, associated with non-Hermitian degeneracies of Eq. (1), can be achieved and made completely reversible in two opposite directions. The underlying physical mechanism is illustrated by considering a lattice of driven cold atoms [25-29] whose probe susceptibility is such that $\chi_{p}(z)=-\chi_{p}^{*}(-z)$ with loss and no gain, which is clearly not a $P T$-symmetric case [30,31]. We engineer a far-detuned dressing field so as to induce a spatially modulated frequency shift along the lattice axis in quadrature with respect to the atomic density (see Fig. 1). Our analysis may also be adapted to defect centers in solids [32]. Consequently, we may observe unidirectional reflectionlessness at a non-Hermitian degeneracy of the scattering matrix. It is of special interest that the unidirectional vanishing probe reflectivity can be reverted by changing detuning signs of dressing field components. 
In particular, we consider cold ${ }^{87} \mathrm{Rb}$ atoms loaded into the dipole traps of an optical lattice formed by the retroreflecting red-detuned laser beams of wavelength $\lambda_{o}$ [see Fig. 1(a)]. The resultant atomic lattices have the periodicity $a=0.5 \lambda_{o} / \cos \theta_{o}$ with $\theta_{o}$ being a small angle between the dipole-trap laser beams (which are not shown in Fig. 1) and the lattice axis along $\vec{z}$ [33]. The tightly trapped ${ }^{87} \mathrm{Rb}$ atoms typically exhibit a Gaussian density distribution $N_{j}(z)=N_{0} \exp \left[-\left(z-z_{j}\right)^{2} / d^{2}\right]$ in each period with $d$ being the $1 / e$ half width and $z_{j}$ the $j$ th lattice center. The Gaussian width $d$ depends on the trapping depth $U_{0}$ of dipole potentials and the average temperature $T$ of trapped atoms via $d=\left(\lambda_{o} \sqrt{\kappa_{B} T}\right) /\left(2 \pi \sqrt{2 U_{0}}\right)$ [27]. Such periodically distributed atoms are driven into the four-level $N$ configuration by three coherent fields of frequencies (amplitudes) $\omega_{p}\left(\mathbf{E}_{p}\right), \omega_{c}\left(\mathbf{E}_{c}\right)$, and $\omega_{d}\left(\mathbf{E}_{d}\right)$ [see Fig. 1(b)]. The weak probe field $\omega_{p}$, moderate coupling field $\omega_{c}$, and strong dressing field $\omega_{d}$ interact, respectively, with transitions $|1\rangle \leftrightarrow|3\rangle,|2\rangle \leftrightarrow|3\rangle$, and $|2\rangle \leftrightarrow|4\rangle$. The corresponding detunings (Rabi frequencies) are defined as $\Delta_{p}=\omega_{p}-\omega_{31}, \Delta_{c}=\omega_{c}-\omega_{32}$, and $\Delta_{d}=$ $\omega_{d}-\omega_{42} \quad\left(\Omega_{p}=\mathbf{E}_{p} \mathbf{d}_{13} / 2 \hbar, \quad \Omega_{c}=\mathbf{E}_{c} \mathbf{d}_{23} / 2 \hbar, \quad\right.$ and $\left.\Omega_{d}=\mathbf{E}_{d} \mathbf{d}_{24} / 2 \hbar\right)$ with $\omega_{i j}$ being atomic transition frequencies and $\mathbf{d}_{i j}$ being electric dipole moments.
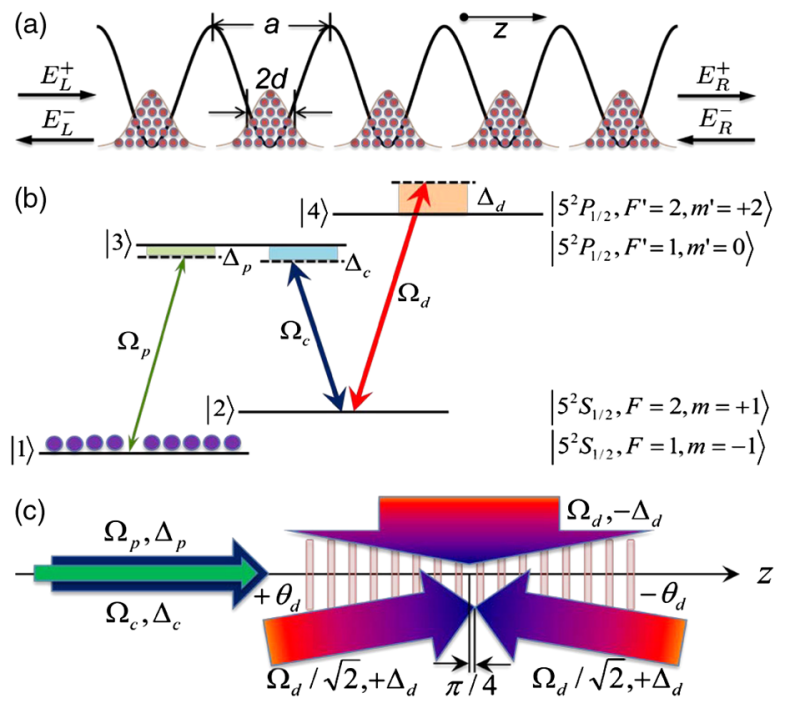

FIG. 1 (color online). (a) An ensemble of cold ${ }^{87} \mathrm{Rb}$ atoms is loaded into a 1D optical lattice of dipole traps. (b) These atoms interact with a near-resonant probe $\left(\Omega_{p}\right)$ field, a near-resonant coupling $\left(\Omega_{c}\right)$ field, and a far-detuned dressing $\left(\Omega_{d}\right)$ field. (c) Probe and coupling fields travel along the lattice $z$ axis with $\sigma^{+}$and $\sigma^{-}$polarizations, respectively. The dressing field has instead two components nearly counterpropagating at a very small angular offset $\pm \theta_{d}$, both with $\sigma^{+}$polarization, to give a spatial modulation of the light shift $\delta_{d s}(z)$ of transition $|2\rangle \leftrightarrow|4\rangle$, and a third component propagating along the $x$ direction and linearly polarized along $y$ to provide a light shift that is uniform in space (see text).
Within the rotating-wave and electric-dipole approximations, the equations of motion for the density matrix elements $\rho_{i j}$ are obtained as usual. By setting $\rho_{11} \simeq 1$ in the weak probe limit and assuming $\gamma_{12} \ll \gamma_{13} \simeq \gamma_{14}=\gamma$ for the dephasing rates $\gamma_{i j}$, we can obtain

$$
\rho_{31}=\frac{i \Omega_{p}\left[\gamma_{12}-i\left(\Delta_{p}-\delta_{d 0}\right)\right]}{\Omega_{c}^{2}+\left(\gamma-i \Delta_{p}\right)\left[\gamma_{12}-i\left(\Delta_{p}-\delta_{d 0}\right)\right]}
$$

with $\delta_{d 0}=-\Omega_{d}^{2} / \Delta_{d}$ being the dynamic frequency shift of the $|2\rangle-|4\rangle$ transition. In deriving Eq. (2), we have also set $\Delta_{c}=0, \Delta_{p} \lesssim \gamma, \Delta_{d} \gg \Omega_{d}$, and $\Delta_{d} \gg \gamma$. A probe susceptibility $\chi_{p}(z)$ with its real (imaginary) part being an even (odd) function of the lattice position $z$ can be implemented by spatially modulating at least two critical parameters of atom-field interaction in distinct elaborate ways [17-19]. Since the atomic density distribution $N_{j}(z)$ is here an even function of the lattice position $z$, we now try to engineer the dynamic frequency shift as an odd function of the lattice position. That is, we take the dressing field to have three components with $\sigma^{+}$polarization [see Fig. 1(c)] described, respectively, by $E_{d} \exp \left[+i k_{d} x-i\left(\omega_{42}-\Delta_{d}\right) t\right]$, $\left(E_{d} / \sqrt{2}\right) \exp \left[+i\left(k_{d}^{\prime} z-\pi / 4\right)-i\left(\omega_{42}+\Delta_{d}\right) t\right]$, and $\left(E_{d} / \sqrt{2}\right)$ $\exp \left[-i\left(k_{d}^{\prime} z-\pi / 4\right)-i\left(\omega_{42}+\Delta_{d}\right) t\right] \quad$ with $\quad k_{d}=2 \pi / \lambda_{d}$, $k_{d}^{\prime}=k_{d} \cos \theta_{d}$, and $\theta_{d}$ being a small angle between the latter two components and the lattice axis $\vec{z}$ [34]. Then the probe susceptibility in the $j$ th period of our atomic lattices reads

$$
\chi_{p_{j}}(z)=\frac{i \alpha_{j}(z)\left\{\gamma_{12}-i\left[\Delta_{p}-\delta_{d s}(z)\right]\right\}}{\Omega_{c}^{2}+\left(\gamma-i \Delta_{p}\right)\left\{\gamma_{12}-i\left[\Delta_{p}-\delta_{d s}(z)\right]\right\}}
$$

with $\alpha_{j}(z)=N_{j}(z) d_{13}^{2} / 2 \varepsilon_{0} \hbar, \quad \delta_{d s}(z)=\delta_{d 0} \sin (\beta z)$, and $\beta=2 k_{d}^{\prime}$. We can attain $\beta=2 \pi / a$ by accurately modulating the two angles $\theta_{o}$ and $\theta_{d}$ even if $\lambda_{d} \neq \lambda_{o}$.

In Fig. 2, we calculate imaginary and real parts of the probe susceptibility $\chi_{p j}(z)$ as a function of lattice position and probe detuning in panels (a) and (b), of lattice position at probe resonance in panels (c) and (d). For a given probe detuning $\Delta_{p}, \operatorname{Im}\left[\chi_{p j}(z)\right]$ and $\operatorname{Re}\left[\chi_{p j}(z)\right]$ are modulated in distinct ways along the lattice axis. At $\Delta_{p}=0$, in particular, our atomic lattices exhibit the antisymmetry described by $\chi_{p j}(+z)=-\chi_{p j}^{*}(-z)$. With the increasing of $\left|\Delta_{p}\right|$, however, this peculiar antisymmetry is gradually lifted as shown in Figs. 2(a) and 2(b). Most importantly, it is possible to control the ratio between maximum amplitudes of $\operatorname{Im}\left[\chi_{p j}(+z)\right]$ and $\operatorname{Re}\left[\chi_{p j}(+z)\right]$ by changing Rabi frequency $\Omega_{d}$ (i.e., dynamic shift $\delta_{d 0}$ ) [see Figs. 2(c) and 2(d)]. This is crucial in achieving non-Hermitian degeneracies [35] and the associated unidirectional reflectionless propagation, as discussed below.

The probe susceptibility in Eq. (3) has a lot of components oscillating at $\exp [ \pm i m \beta z]$ with $m \in\{0,1,2, \ldots\}$, but only its zero and first-order components are responsible for 

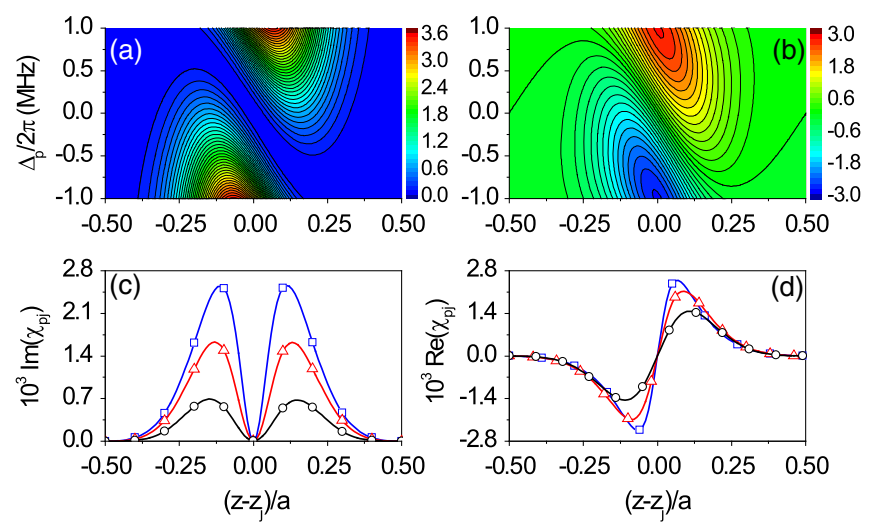

FIG. 2 (color online). Plots of $10^{3} \operatorname{Im}\left(\chi_{p j}\right)$ and $10^{3} \operatorname{Re}\left(\chi_{p j}\right)$ [(a), (b)] vs lattice position $\left(z-z_{j}\right) / a$ and probe detuning $\Delta_{p} / 2 \pi$ with $\gamma_{12} / 2 \pi=2.0 \mathrm{kHz}$ (increasing $\gamma_{12} / 2 \pi$ up to $20 \mathrm{kHz}$ is immaterial, while for larger values the absorption at each trap center starts to increase somewhat, without qualitatively affecting our results), $\gamma / 2 \pi=3.0 \mathrm{MHz}, \Omega_{c} / 2 \pi=2.0 \mathrm{MHz}$, $\Omega_{d} / 2 \pi=16 \mathrm{MHz}, \quad \Delta_{c}=0, \quad \Delta_{d} / 2 \pi=300 \mathrm{MHz}, \quad a=5 d \simeq$ $400 \mathrm{~nm}, \quad d_{13}=2.0 \times 10^{-29} \mathrm{Cm}$, and $N_{0}=0.5 \times 10^{12} \mathrm{~cm}^{-3}$. [(c), (d)] Same as [(a), (b)] plotted at $\Delta_{p}=0$ for $\Omega_{d} / 2 \pi=$ $16 \mathrm{MHz}$ (black circles), $22 \mathrm{MHz}$ (red triangles), $28 \mathrm{MHz}$ (blue squares).

the dynamic propagation of a probe field. In the case of $\gamma_{12} \rightarrow 0$ and $\Delta_{p}=0$, we can obtain

$$
\begin{aligned}
\chi_{p}(z) \sim & i \chi_{p 0}+i \chi_{p a} \cos \beta z-\chi_{p b} \sin \beta z \\
= & \frac{\alpha_{0}(0) x}{\gamma}\left[f_{p b}\left(b_{0}-b_{2}\right)(i x-\sin \beta z)\right. \\
& \left.+\frac{i x}{2} f_{p a}\left(b_{1}-b_{3}\right) \cos \beta z\right]
\end{aligned}
$$

with $x=\gamma \delta_{d 0} / \Omega_{c}^{2}$ and $b_{n}=\sqrt{\pi}(d / a) \exp \left[-(n \pi d / a)^{2}\right]$ while $\chi_{p 0}=x_{p b}$. In Fig. 3, we plot the numerically calculated coefficients $f_{p a}$ and $f_{p b}$ (approximately equal to each other), along with $\chi_{p a}$ and $\chi_{p b}$, as a function of dynamic shift. By varying $\delta_{d 0}$ we can balance the first-order modulation amplitudes of real and imaginary parts of the probe susceptibility, i.e., $\chi_{p a}=\chi_{p b}$, which is crucial to attain non-Hermitian degeneracies (see below). This occurs at $\delta_{d 0} / 2 \pi \simeq 2.58 \mathrm{MHz}$ for $d=a / 5$ [see Fig. 3(b)] while at $\delta_{d 0} / 2 \pi \simeq 1.68 \mathrm{MHz}$ for $d=a / 10$ [not shown].

The property of high-contrast asymmetric reflectivity can be examined either by incorporating Eq. (3) into a transfer-matrix calculation [39] or by incorporating Eq. (4) into a coupled-mode analysis as shown below. The former method allows exact full numerical solutions with arbitrary probe detunings $\Delta_{p} \neq 0$, while the latter method is favorable for getting physical insights in the relevant case of vanishing probe detuning $\Delta_{p}=0$. We present in Fig. 4 calculations of both left and right probe reflectivities as a function of dynamic shift and probe detuning [Figs. 4(a) and 4(b)], of dynamic shift at probe resonance [Figs. 4(c)
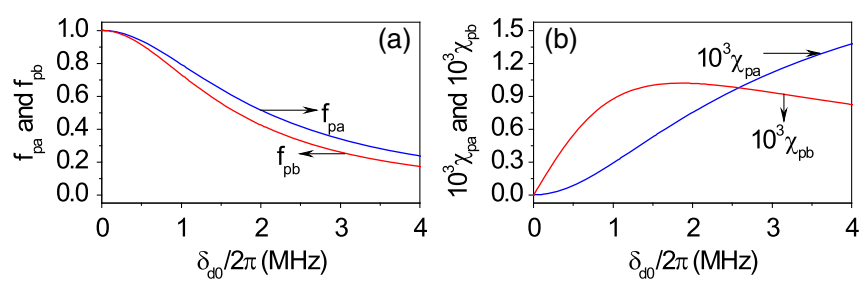

FIG. 3 (color online). Plots of $f_{p a}$ and $f_{p b}(\mathrm{a}) ; \chi_{p a}$ and $\chi_{p b}$ (b) vs dynamic shift $\delta_{d 0} / 2 \pi$ attained from Eq. (4) with the same parameters as in Fig. 2 except $\Delta_{p}=0$.

and 4(d)] with two insets showing the corresponding reciprocal transmissivity spectra $T=T_{L}=T_{R}$. It is clear that we have $R_{L}\left(\delta_{d 0}\right)=R_{R}\left(-\delta_{d 0}\right)$ for a given value of detuning $\Delta_{p}$, and there is a remarkable difference between $R_{L}\left(\delta_{d 0}\right)$ and $R_{R}\left(\delta_{d 0}\right)$ when $\left|\Delta_{p}\right|$ is not too large [see Figs. 4(a) and 4(b)]. As an example, we show in Figs. 4(c) and $4(\mathrm{~d})$ that a vanishing probe reflectivity $\left(R_{L}\right.$ or $\left.R_{R}\right)$ occurs at the exceptional point of $\delta_{d 0} / 2 \pi \simeq \pm 1.68 \mathrm{MHz}$ $\left(\delta_{d 0} / 2 \pi \simeq \pm 2.58 \mathrm{MHz}\right)$ for a smaller (larger) Gaussian width $d=a / 10 \quad(d=a / 5)$. The reflectivity contrast $C_{R}=\left|\left(R_{R}-R_{L}\right) /\left(R_{R}+R_{L}\right)\right|$, sensitive to $\Delta_{p}$ and $\delta_{d 0}$, quickly decreases from $100 \%$ to $80 \%$, e.g., at the exceptional point of $\delta_{d 0} / 2 \pi \simeq \pm 1.68 \mathrm{MHz}(a=10 d)$ when $\left|\Delta_{p} / 2 \pi\right|$ is increased from 0 to $0.15 \mathrm{MHz}$.

Within the framework of non-Hermitian physics it is possible that a pair of eigenvalues become degenerate and the corresponding eigenvectors coalesce into a single state (non-Hermitian degeneracy). In Fig. 5, we plot eigenvalues $\lambda_{s}^{ \pm}=t \pm \sqrt{r_{L} r_{R}}$ of the matrix $S$, which exhibit the typical topology of a non-Hermitian degeneracy and, in particular, a transition from complex values to real values at $\delta_{d 0} / 2 \pi \simeq$ $1.68 \mathrm{MHz}$ along the branch cut at $\Delta_{p}=0$. At the transition point, $r_{L}$ becomes exactly zero [See Fig. 4(c)] and the matrix
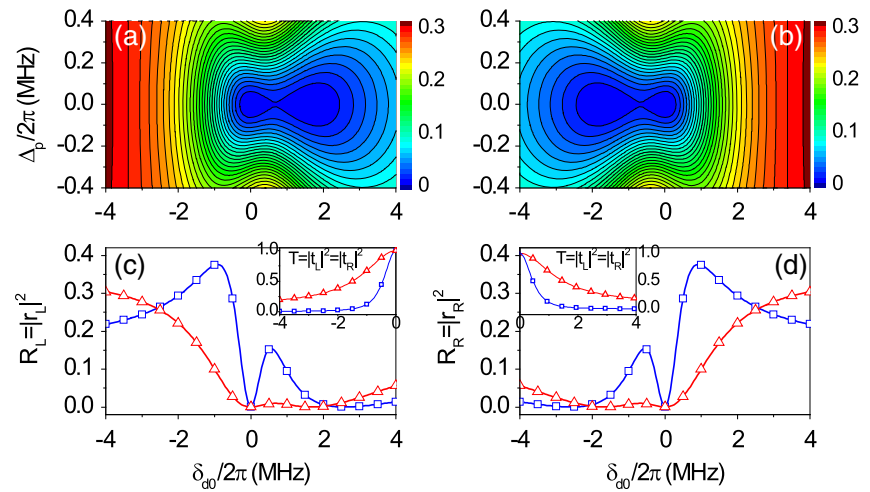

FIG. 4 (color online). Plots of $R_{L}$ (a) and $R_{R}$ (b) vs dynamic shift $\delta_{d 0} / 2 \pi$ and probe detuning $\Delta_{p} / 2 \pi$ for atomic lattices of length $L=0.6 \mathrm{~mm}$ with the same parameters as in Fig. 2 except $d=a / 10$. Plots of $R_{L}$ (c) and $R_{R}$ (d) vs dynamic shift $\delta_{d 0} / 2 \pi$ with the same parameters as in (a) and (b) except $\Delta_{p}=0$ and $d=a / 10$ for red curves with triangles; $d=a / 5$ for blue curves with squares. The two insets show the corresponding reciprocal transmissivity $T=T_{L}=T_{R}$. 

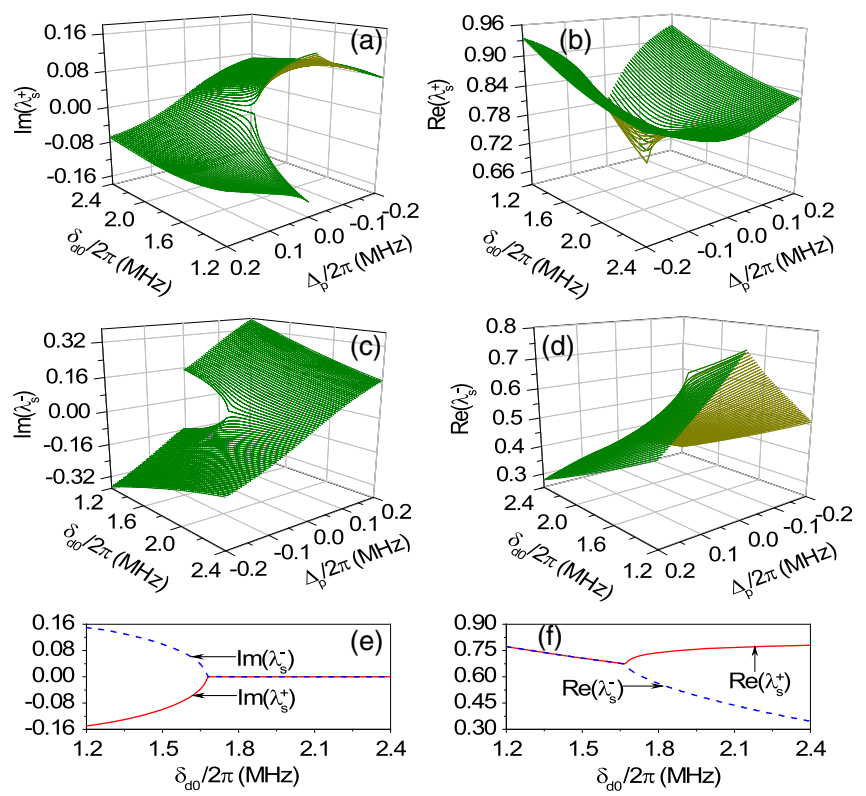

FIG. 5 (color online). Real and imaginary parts of $\lambda_{s}^{ \pm}[(\mathrm{a})-(\mathrm{d})]$ vs dynamic shift $\delta_{d 0} / 2 \pi$ and probe detuning $\Delta_{p} / 2 \pi$. Parameters are the same as in Fig. 2 except $d=a / 10$ and $L=0.6 \mathrm{~mm}$. Axes are oppositely oriented to better display the topology of the nonHermitian degeneracy at $\Delta_{p}=0$ and $\delta_{d 0} / 2 \pi \simeq 1.68 \mathrm{MHz}$. $\operatorname{Im}\left(\lambda_{s}^{ \pm}\right)$and $\operatorname{Re}\left(\lambda_{s}^{ \pm}\right)[(\mathrm{e}),(\mathrm{f})]$ vs dynamic shift $\delta_{d 0} / 2 \pi$ at $\Delta_{p}=0$.

$S$ (un-normalized) eigenvectors $\left|\varphi_{ \pm}\right\rangle=\left( \pm \sqrt{r_{L} / r_{R}}, 1\right)^{T}$ coalesce into the (only) state $\left|\varphi_{d}\right\rangle=(0,1)^{T}$ so that $r_{L}=0$ represents a non-Hermitian degeneracy. Since the eigenvectors $\left\{\left|\varphi_{+}\right\rangle,\left|\varphi_{-}\right\rangle\right\}$are not orthogonal and coalesce into the (only) state $\left|\varphi_{d}\right\rangle$ at degeneracy $\left(r_{L}=0\right.$ ), they no longer form a complete basis. As commonly done with the non-Hermitian matrices, one needs to introduce the left eigenvectors $\left\langle\tilde{\varphi}_{ \pm}\right|=\left( \pm \sqrt{r_{R} / r_{L}}, 1\right)$ [40]. Left and right eigenvectors (for distinct eigenvalues $\lambda_{s}^{ \pm}$) are now orthogonal $\left\langle\tilde{\varphi}_{ \pm} \mid \varphi_{\mp}\right\rangle=0$, yet at degeneracy they are self-orthogonal, i.e., $\left\langle\tilde{\varphi}_{d} \mid \varphi_{d}\right\rangle=0$, which reflects the fact that the matrix $S$ can no longer be diagonalized. Despite the fact that selforthogonality is difficult to observe [41], we note that the state $\left|\varphi_{d}\right\rangle$ corresponds, through Eq. (1), to a well defined physical scattering state with $E_{L}^{-}=E_{R}^{-}=0, E_{R}^{+}=t$, and $E_{L}^{+}=1$, i.e., a state with complete one-way reflectionless propagation for light coming from the left. An analogous discussion holds for the other non-Hermitian degeneracy $r_{R}=0$ [see Fig. 4(d)].

At $\Delta_{p}=0$, analytical coupled-mode equations can be derived for the probe field $E_{p}(z)=E_{f}(z) \exp [+i \beta z / 2]+$ $E_{b}(z) \exp [-i \beta z / 2]$ using the zero- and first-order components of $\chi_{p}(z)$ from Eq. (4) in the Helmholtz equation; proceeding as usual, we obtain

$$
\begin{aligned}
& \partial_{z} E_{f}=-\eta_{1}\left[x E_{f}+\left(1+x \eta_{2} / 2\right) E_{b}\right], \\
& \partial_{z} E_{b}=+\eta_{1}\left[x E_{b}-\left(1-x \eta_{2} / 2\right) E_{f}\right]
\end{aligned}
$$

with $\eta_{1}=\beta \chi_{p b} / 8$ and $\eta_{2}=(2 / x)\left(\chi_{p a} / \chi_{p b}\right)$. The forward $\left(E_{f}\right)$ and backward $\left(E_{b}\right)$ components experience different cross feedback if $x \eta_{2} \neq 0\left(\chi_{p a} \neq 0\right)$. The non-Hermitian degeneracy corresponds, in fact, to $x \eta_{2}= \pm 2$ whereby one component suffers only self absorption, i.e., loses all feedback from the other component.

Using boundary conditions $E_{f}(0)=E_{L 0}$ and $E_{b}(L)=0$ as well as $E_{f}(0)=0$ and $E_{b}(L)=E_{R 0}$, we can determine from Eqs. (5) the nonreciprocal reflectivities

$$
R_{R, L}=\left|\frac{ \pm \eta_{1}\left(1 \pm x \eta_{2} / 2\right)\left(e^{+\lambda L}-e^{-\lambda L}\right)}{\left(\lambda+x \eta_{1}\right) e^{+\lambda L}+\left(\lambda-x \eta_{1}\right) e^{-\lambda L}}\right|^{2}
$$

with propagation constant $\lambda= \pm \eta_{1} \sqrt{x^{2}\left(1-\eta_{2}^{2} / 4\right)+1}$. The reciprocal transmissivity $T=T_{R}=T_{L}$ has a similar expression in which the numerator is $2 \lambda$ instead. Thus, $R_{L}$ will be different from $R_{R}$ if we have $x \eta_{2} \neq 0$. At the exceptional point $x=2 / \eta_{2}$, in particular, $\lambda= \pm x \eta_{1}$, $T=e^{-2 x \eta_{1} L}, \quad R_{L}=0$, and $R_{R}=\left[e^{-2 x \eta_{1} L}-1\right]^{2} / x^{2}$. For sufficiently long and dense atomic lattices, $T \rightarrow 0$ and $\quad R_{R} \rightarrow 1 / x^{2}=\left[f_{p a}\left(b_{1}-b_{3}\right)\right]^{2} /\left[2 f_{p b}\left(b_{0}-b_{2}\right)\right]^{2} \simeq$ $\left[\left(b_{1}-b_{3}\right) /\left(b_{0}-b_{2}\right)\right]^{2} / 4$ so that a smaller $d / a$ is desired for attaining a larger $R_{R}$ with $R_{L}=0$ and $T \rightarrow 0$. Modulating the dressing field detuning from $+\Delta_{d}$ to $-\Delta_{d}$, we obtain instead $T \rightarrow 0, R_{R}=0$, and $R_{L} \simeq$ $\left[\left(b_{1}-b_{3}\right) /\left(b_{0}-b_{2}\right)\right]^{2} / 4$. These analytical predictions based on the coupled-mode theory are in excellent agreement with the numerical calculations based on the transfermatrix approach.

In summary, full unidirectional reflectionless behavior of light transport in a 1D scattering medium occurs at nonHermitian degeneracies of the scattering matrix $S$. Cold atomic lattices are shown to be a viable platform to explore the intriguing structures of these degeneracies, intrinsic to non-Hermitian optics even without gain.

We would like to thank Simon Horsley for fruitful discussions. This work is supported by NSFC (Grants No. 11174110 and No. 61378094) and NBRP (Grant No. 2011CB921603) of China. J.-H. W. acknowledges the hospitality of Scuola Normale Superiore at Pisa.

[1] E. Istrate and E. H. Sargent, Rev. Mod. Phys. 78, 455 (2006).

[2] D. R. Smith and N. Kroll, Phys. Rev. Lett. 85, 2933 (2000).

[3] A. Hache and M. Bourgeois, Appl. Phys. Lett. 77, 4089 (2000).

[4] X.-Y. Hu, Y.-H. Liu, J. Tian, B.-Y. Cheng, and D.-Z. Zhang, Appl. Phys. Lett. 86, 121102 (2005).

[5] J. D. Teufel, T. Donner, M. A. Castellanos-Beltran, J. W. Harlow, and K. W. Lehnert, Nat. Nanotechnol. 4, 820 (2009).

[6] X. Zhang and Z.-W. Liu, Nat. Mater. 7, 435 (2008).

[7] Z. Lin, H. Ramezani, T. Eichelkraut, T. Kottos, H. Cao, and D. N. Christodoulides, Phys. Rev. Lett. 106, 213901 (2011). 
[8] L. Feng, Y.-L. Xu, W. S. Fegadolli, M.-H. Lu, J. E. B. Oliveira, V. R. Almeida, Y.-F. Chen, and A. Scherer, Nat. Mater. 12, 108 (2012).

[9] S. Longhi, Phys. Rev. Lett. 103, 123601 (2009).

[10] C. E. Ruter, K. G. Makris, R. EI-Ganainy, D. N. Christodoulides, M. Segev, and D. Kip, Nat. Phys. 6, 192 (2010).

[11] A. Regensburger, C. Bersch, M.-A. Miri, G. Onishchukov, D. N. Christodoulides, and U. Peschel, Nature (London) 488, 167 (2012).

[12] Y.-D. Chong, L. Ge, H. Cao, and A. D. Stone, Phys. Rev. Lett. 105, 053901 (2010).

[13] S. Longhi, Phys. Rev. A 82, 031801(R) (2010).

[14] V. V. Konotop, V. S. Shchesnovich, and D. A. Zezyulin, Phys. Lett. A 376, 2750 (2012).

[15] M.-A. Miri, P. LiKamWa, and D. N. Christodoulides, Opt. Lett. 37, 764 (2012).

[16] M.-A. Miri, A. B. Aceves, T. Kottos, V. Kovanis, and D. N. Christodoulides, Phys. Rev. A 86, 033801 (2012).

[17] C. Hang, G.-X. Huang, and V. V. Konotop, Phys. Rev. Lett. 110, 083604 (2013).

[18] J.-T. Sheng, M.-A. Miri, D. N. Christodoulides, and M. Xiao, Phys. Rev. A 88, 041803(R) (2013).

[19] H.-J. Li, J.-P. Dou, and G. Huang, Opt. Express 21, 32053 (2013).

[20] S. A. R. Horsley, J.-H. Wu, M. Artoni, and G. C. La Rocca, Phys. Rev. Lett. 110, 223602 (2013).

[21] D.-W. Wang, H.-T. Zhou, M.-J. Guo, J.-X. Zhang, J. Evers, and S.-Y. Zhu, Phys. Rev. Lett. 110, 093901 (2013).

[22] C. M. Bender and S. Boettcher, Phys. Rev. Lett. 80, 5243 (1998).

[23] M. Berry, Czech. J. Phys. 54, 1039 (2004).

[24] A. Mostafazadeh, Phys. Rev. Lett. 102, 220402 (2009).

[25] I. Bloch, Nat. Phys. 1, 23 (2005).

[26] A. Schilke, C. Zimmermann, P. W. Courteille, and W. Guerin, Phys. Rev. Lett. 106, 223903 (2011).

[27] A. Schilke, C. Zimmermann, and W. Guerin, Phys. Rev. A 86, 023809 (2012).

[28] J.-H. Wu, S. A. R. Horsley, M. Artoni, and G. C. La Rocca, Light Sci. Appl. 2, e54 (2013).

[29] J.-H. Wu, M. Artoni, and G. C. La Rocca, Phys. Rev. A 88, 043823 (2013).

[30] Non $P T$-symmetric models with an antisymmetric index $n(z)=-n^{*}(-z)$ have recently been considered [31], clearly at variance with the antisymmetry of our susceptibility $\chi(z)=-\chi^{*}(-z)$ [see Figs. 2(c) and 2(d)]. Those models further require impractical balances of positive and negative index materials.

[31] L. Ge and H.E. Türeci, Phys. Rev. A 88, 053810 (2013).

[32] J.-H. Wu, G. C. La Rocca, and M. Artoni, Phys. Rev. B 77, 113106 (2008).

[33] Employing far-off resonant dipole trap laser beams with a frequency between the $D 1$ and $D 2$ lines the angle $\theta_{o}$ is of several degrees.

[34] The $\sigma^{-}$polarized component of dressing field propagating along $x$ couples to the $|2\rangle-|3\rangle$ transition producing a spatially uniform light shift, which can be included in $\Delta_{c}$, whereas the light shift of the $|1\rangle-|3\rangle$ transition due to the $\sigma^{+}$polarized components of dressing field is negligible, as they are far detuned from this transition.

[35] Non-Hermitian degeneracies are also known as "exceptional points" [36], whose nontrivial branch-point structure for the relevant Hamiltonian spectrum has been the subject of extended theoretical investigations [23,37,38].

[36] T. Kato, Perturbation Theory for Linear Operators (Springer-Verlag, Berlin, 1980).

[37] W. D. Heiss, Czech. J. Phys. 54, 1091 (2004).

[38] A. Ghatak, R. D. R. Mandal, and B. P. Mandal, Ann. Phys. (Amsterdam) 336, 540 (2013).

[39] M. Artoni, G. C. La Rocca, and F. Bassani, Phys. Rev. E 72, 046604 (2005).

[40] Left eigenvectors satisfy $\left\langle\tilde{\varphi}_{ \pm}\right|\left(S-\lambda_{ \pm}\right)=0$, unlike the usual (right) column vectors satisfying $\left(S-\lambda_{ \pm}\right)\left|\varphi_{ \pm}\right\rangle=0$.

[41] Self-orthogonal phenomena extensively studied in nuclear, atomic, and molecular physics and especially in the context of $P T$-symmetric Hamiltonians [42] often bear little physical relevance as they are not easily accessible to experiments [43-46].

[42] J. Okolowicz, M. Ploszajczak, and I. Rotter, Phys. Rep. 374, 271 (2003).

[43] C. Dembowski, H. D. Graf, H. L. Harney, A. Heine, W. D. Heiss, H. Rehfeld, and A. Richter, Phys. Rev. Lett. 86, 787 (2001).

[44] Y. Choi, S. Kang, S. Lim, W. Kim, J.-R. Kim, J.-H. Lee, and K. An, Phys. Rev. Lett. 104, 153601 (2010).

[45] E. Narevicius and N. Moiseyev, Phys. Rev. Lett. 84, 1681 (2000).

[46] R. Lefebvre, O. Atabek, M. Sindelka, and N. Moiseyev, Phys. Rev. Lett. 103, 123003 (2009). 